\title{
HISTOPATHOLOGICAL PATTERN IN LUNG AUTOPSY IN GOVERNMENT MEDICAL COLLEGE JAMMU
}

\author{
Shweta1, Deepti Mahajan², Vidhu Mahajan³, P. Angmo
}

${ }_{1}^{1}$ Post Graduate, Department of Pathology, GMC Jammu, India. ${ }^{2}$ Assitant Professor, Department of Pathology, GMC Jammu, India. ${ }^{3}$ Post Graduate, Department of Pathology, ASCOMS Jammu, India. ${ }^{4}$ Associate Professor, Department Pathology, GMC Jammu, India.

ABSTRACT: The lungs are involved in various kinds of inflammatory, neoplastic and other lesions, but they are secondarily involved in almost all form of terminal diseases. Autopsy has a role in the development of new understanding of old diseases and provision of opportunity to discover new diseases.

AIM: To present the pulmonary histopathological pattern identified in autopsy.

METHODOLOGY: This is a prospective study done in the Department of Pathology, GMC Jammu over a period of 1 years from 2013 to 2014. Materials for study consisted of lungs from 150 cases from medico-legal autopsies received during this period. Gross features were recorded and microscopic diagnosis were made. Results: Among the 150 lung specimens from medicolegal autopsies, 132 were males and 18 were females Findings of lung disease were seen in 138 cases, of which pulmonary edema in 39 cases, DAD in 32 cases Acute Pulmonary congestion in 26 cases, chronic ILD in 12 cases, acute pneumonia in 8 cases, Tuberculosis in 5 cases. CONCLUSION: Histomorphological study of lung in medicolegal autopsies may quite often reveal some natural disease and its relative contribution towards death.

KEYWORDS: Lung Autopsy, Medicolegal Autopsies, Histomorphological Pattern.

HOW TO CITE THIS ARTICLE: Shweta, Deepti Mahajan, Vidhu Mahajan, P. Angmo. "Histopathological Pattern in Lung Autopsy in Government Medical College Jammu". Journal of Evolution of Medical and Dental Sciences 2015; Vol. 4, Issue 91, November 12; Page: 15694-15696, DOI: $10.14260 /$ jemds/2015/2259.

INTRODUCTION: Autopsy is an important and most useful way to find out the condition of internal organs. An autopsy may be required in deaths that may have medical and legal issues. In addition to ascertain clinico-pathological differences, autopsy has a role in the development of new understanding of old diseases and provision of opportunity to discover new diseases. Medicolegal autopsy or postmortem examination is a special type of scientific examination of dead body carried out under law of state mainly for the protection of the citizens and to assist the identification and prosecution of the guilty in case of unnatural death. The principal pulmonary histopathological alterations seen in most of the autopsy cases are: Diffuse alveolar damage (DAD), Pulmonary edema, lymphocytic interstitial pneumonia (LIP), alveolar hemorrhage, Organising pneumonia and Tuberculosis. (Alexander de Matos et al, 2008).[1] (Hijoth L et al, 1995).[2] Since the lungs are involved in various kinds of inflammatory, neoplastic and other lesions, but they are secondarily involved in almost all form of terminal diseases. The aim of the present study was to present the pulmonary histopathological alterations identified in autopsies.

LITERATURE SURVEY: Males were more affected with lung lesions in studies conducted by Bal MS et al.[3] In an autopsy study by Bal et al, lungs were involved in majority of the cases $(77.33 \%)$ of the cases while primary lesions leading to death were only $33.66 \%$ cases (2008) .

Financial or Other, Competing Interest: None.

Submission 23-10-2015, Peer Review 24-10-2015,

Acceptance 03-11-2015, Published 11-11-2015.

Corresponding Author:

Dr. Shweta,

Department of Pathology,

GMC, Jammu.

E-mail: nipunkalsotra@yahoo.com

DOI:10.14260/jemds/2015/2259.
Tuberculosis was the major cause of death in study by Tariq MT et al (2007).[4]

MATERIALS AND METHODS: This is a retrospective study done in the Department of Pathology, GMC Jammu over a period of 1 year from 2013 to 2014. Materials for study consisted of lungs from 150 cases (Medico legal and clinical) received in the Department of Pathology during this period.

Inclusion Criteria: The subjects were selected from medico legal autopsies irrespective of cause of death.

Study Design: This study was a non-interventional, Descriptive study.

Data Collection Procedure: Information regarding age and brief history of illness etc were obtained from the request forms.

RESULTS / DISCUSSION: Commonest pathology encountered was pulmonary edema followed by emphysematous change, pneumonias and tuberculosis. Males were more affected than females.

\begin{tabular}{|c|c|c|c|}
\hline & $\begin{array}{c}\text { Total } \\
\text { specimens }\end{array}$ & $\begin{array}{c}\text { Lung } \\
\text { Pathology }\end{array}$ & $\begin{array}{c}\text { Percentage } \\
\text { (\%) }\end{array}$ \\
\hline Male & 132 & 124 & 93.9 \\
\hline Female & 18 & 14 & 77 \\
\hline Total & 150 & $\mathbf{1 3 8}$ & $\mathbf{9 2}$ \\
\hline \multicolumn{3}{|c|}{ Table 1: Prevalance of Lung Pathology } \\
in Males and Females \\
\hline
\end{tabular}




\begin{tabular}{|c|c|c|}
\hline PULMONARY PATTERNS & NO. OF CASES (n) & PERCENTAGE (\%) \\
\hline PULMONARY EDEMA & 39 & 26 \\
\hline DIFFUSE ALVEOLAR DAMAGE & 32 & 21 \\
\hline ACUTE PULMONARY CONGESTION & 26 & 17.3 \\
\hline CHRONIC ILD & 12 & 8 \\
\hline CHRONIC PASSIVE PULMONARY CONGESTION & 9 & 6 \\
\hline ACUTE PNEUMONIA & 8 & 5.3 \\
\hline TUBERCULOSIS & 5 & 3.3 \\
\hline EMPHYSEMA & 2 & 1.3 \\
\hline AUTOLYSED/DECOMPOSED LUNG TISSUE & 5 & 3.3 \\
\hline NORMAL LUNG & 12 & 8 \\
\hline TOTAL & $\mathbf{1 5 0}$ & $\mathbf{1 0 0}$ \\
\hline Table 2: Histopathological Pulmonary Patterns
\end{tabular}

In our study pulmonary edema, diffuse alveolar damage and acute pulmonary congestion, all falling under the preview of acute lung injury, accounted for aprox.

$65 \%$ of the cases, thus forming a major chunk of pulmonary pathology in autopsy cases. These observations are in concordance with those of Bora Ozdemir et al (2012) (Intra alveolar haemorrhage and pulmonary edema together accounting for $71.2 \%$ of the cases), Alexandre de Matos et al in 2008 (Intra alveolar edema, pulmonary congestion and diffuse alveolar damage in $77.7 \%$ of the cases) and Bal et al in 2008 (Lungs showing terminal events like pulmonary edema and ARDS together accounting for $59.3 \%$ of cases).

Acute pneumonia accouned for $5.5 \%$ of cases in the present study which is in concordance with the finding of Bora Ozdemir et al (2012) (8\%) cases, Tahir et al (4\%) cases and Bal et al (9.3) cases. Active tuberculosis with presence of caseating granuloma was the principal pulmonary pathology observed in $3.3 \%$ of cases in the present study. , Alexandre de Matos et al (2008) and V.Selvan et al (2011) have also reported similar prevalence rates $(3.6 \%$ and $2.5 \%$ respectively) for Tuberculous lung at autopsy. Bal et al (2008) observed slightly higher percentage of TB lung cases (8.67\%).

Chronic ILD with or without fibrosis accounted for $8 \%$ of cases in this study. In 5 cases the pattern was cellular consisting of mild to moderate chronic interstitial inflammation containing lymphocytes and few plasma cells in a uniform or patchy distribution resembling the pattern of NSIP. Coexisting patchy fibrosis with fibroblastic foci was seen in 7cases, concomitant with the pattern of usual interstitial pneumonia. 2cases showed accumulation of a large number of macrophages with abundant cytoplasm containing dusty brown pigment in the alveolar space.

(Desquamated interstitial pneumonia). This was associated with mild septal thickening and a chronic inflammatory infiltrate with sprinkling of eosinophils. The pattern suggested smoking related interstitial disease. In the present study the completly autolysed lung tissue account for $3.3 \%$ of cases. Bal et al (2008) observed $12.67 \%$ lung autopsy cases showing complete autolysis.

Acute lung injury, be it, the ubiquitous acute congestion or the more ominous pulmonary edema or diffuse alveolar damage; was the consistent finding in the lung of most of the medicolegal autopsies with death occurring from a wide range of causes ranging from drowning or other form of asphyxia to haemorrhage and shock to burns or septicaemia.

It was also the most common pathological lung pattern observed in death due to proven or presumed cardiac cause as well as in sudden natural deaths. Acute lung injury by itself could be the primary cause of mortality in death due to asphyxia or toxic inhalants.
Among the other lung pathologies observed in the study, acute pneumonia, accounting for 8 cases, was the only primary pulmonary pathology that could have directly resulted in death. The rest of the pulmonary pathological patterns were the associated or incidental findings that may or may not have resulted in death.

In our study Pulmonary edema, Diffuse alveolar damage and Acute pulmonary congestion, all falling under the preview of Acute lung injury, accounted for aprox. $65 \%$ of the cases, thus forming a major chunk of pulmonary pathology in autopsy cases. These observations are in concordance with those of Bora Ozdemir et al (2012).[5] (intra alveolar haemorrhage and pulmonary edema together accounting for $71.2 \%$ of the cases), Alexandre de Matos et al in 2008.[1] (Intra alveolar edema, pulmonary congestion and diffuse alveolar damage in $77.7 \%$ of the cases) and Bal et al in 2008.[3] (Lungs showing terminal events like pulmonary edema and ARDS together accounting for $59.3 \%$ of cases).

Acute pneumonia accouned for $5.5 \%$ of cases in the present study which is in concordance with the finding of Bora Ozdemir et al (2012).[5] (8\%) cases, Tahir et al (4\%). ${ }^{[4]}$ cases and Bal et al (9.3).[3] cases. In another study done by Hjortn et al (1995).[2] on Acute pulmonary infections at autopsy, pneumonia was observed in $23 \%$ cases. Active tuberculosis with presence of caseating granuloma was the principal pulmonary pathology observed in $3.3 \%$ of cases in the present study. , Alexandre de Matos et al (2008).[1] and V.Selvan et al (2011) have also reported similar prevalence rates $3.6 \%$ and $2.5 \%$ respectively) for Tuberculous lung at autopsy. Bal et al(2008).[3] observed slightly higher percentage of TB lung cases $(8.67 \%)$.

Chronic ILD with or without fibrosis accounted for $8 \%$ of cases in this study. In 5 cases the pattern was cellular consisting of mild to moderate chronic interstitial inflammation containing lymphocytes and few plasma cells in a uniform or patchy distribution resembling the pattern of NSIP. Coexisting patchy fibrosis with fibroblastic foci was seen in 7 cases, concomitant with the pattern of Usual interstitial pneumonia. 2 cases showed accumulation of a large number of macrophages with abundant cytoplasm containing dusty brown pigment in the alveolar space. (Desquamated interstitial pneumonia). This was associated with mild septal thickening and a chronic inflammatory infiltrate with sprinkling of eosinophils. The pattern suggested smoking related interstitial disease. In the present study the completly autolysed lung tissue account for $3.3 \%$ of cases. Bal et al 
(2008) observed $12.67 \%$ lung autopsy cases showing complete autolysis.

Acute lung injury, be it, the ubiquitous acute congestion or the more ominous pulmonary edema or diffuse alveolar damage; was the consistent finding in the lung of most of the medicolegal autopsies with death occurring from a wide range of causes ranging from drowning or other form of asphyxia to haemorrhage and shock to burns or septicaemia. It was also the most common pathological lung pattern observed in death due to proven or presumed cardiac cause as well as in sudden natural deaths. Acute lung injury by itself could be the primary cause of mortality in death due to asphyxia or toxic inhalants. Among the other lung pathologies observed in the study, Acute pneumonia, accounting for 8 cases, was the only primary pulmonary pathology that could have directly resulted in death. The rest of the pulmonary pathological patterns were the associated or incidental findings that may or may not have resulted in death.

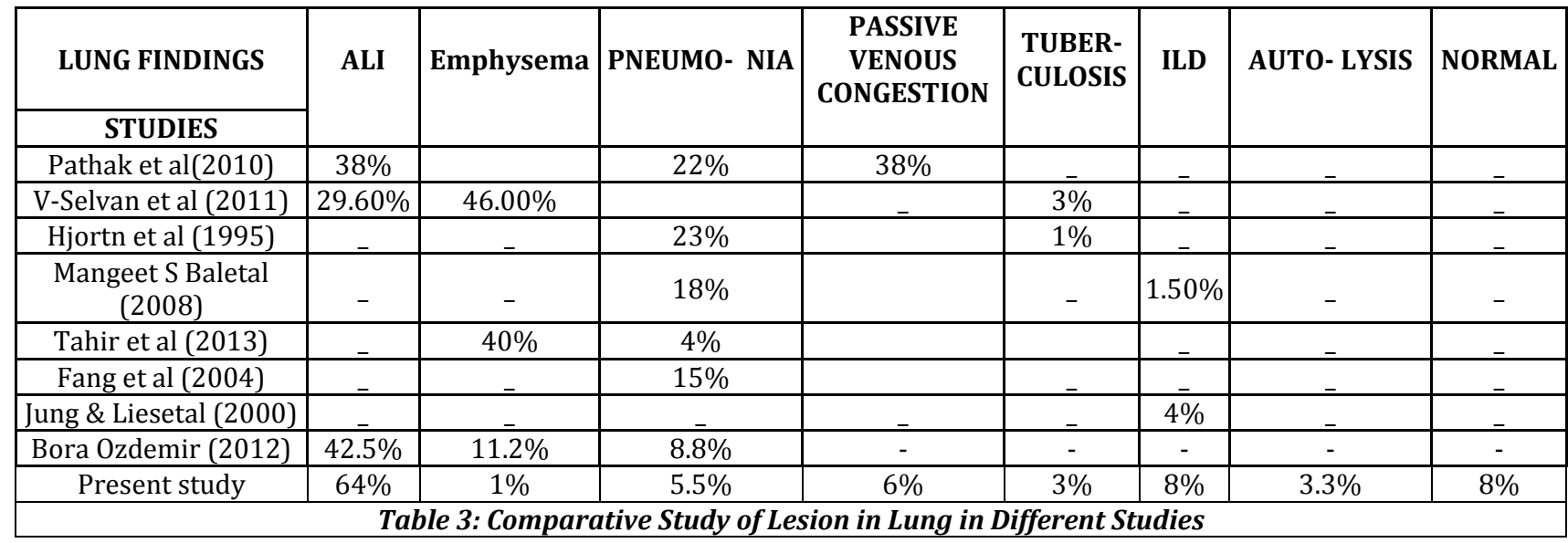

This prospective autopsy study is on a moderate sample. Though lacking in clinical correlation in all cases, gives an indication of lesions of lung at our institution.

CONCLUSION: Autopsy study is of great value in improving the vision and diagnostic setup for better clinical assessment. Thus the histopathological examination of lungs is vital in establishing the cause of death if it is unknown or in confirming an uncertain ante mortem diagnosis.

FUTURE SCOPE: Though technology in diagnostics have advanced, autopsy still serves as a supportive and educative tool in identifying and establishing the underlying cause of death. Hence its importance must be understood, since there has to be newer approaches for old diseases and scope for detection of newer diseases.

\section{REFERENCES:}

1. Alexandre de Matos Soeiro, Edwin Roger Parra, Mauro Canzian et al. Pulmonary histopathological alterations in patients with acute respiratory failure: an autopsy study.. J. bras. Pneumol, 2008; 34: [2].
2. Hjorth L, Jensen HS and Noer H. Acute pulmonary infections at autopsy. A study of clinical and macroscopic diagnoses at autopsy compared with microscopic autopsy findings. Ugeskr Laeger, 1995 ; 157(49): [6873-6].

3. Manjit S Bal, P.S. Sethi, Anil K. Suri, et al. Histopathological pattern in lung autopsy. JPAFMAT, 2008: [8].

4. Tariq Mahmood Tahir, fakeha Rehman, Sadia Anwar et al. Pattern of pulmonary morphological lesions seen at autopsy. Biomedical, 2013; 29: [64].

5. Bora Ozdemir1, Osman Celbis, Rezzan Onal et al .Multiple Organ Pathologies Underlying in Sudden Natural Deaths. Medicine Science, 2012;1(1):[13-26]. 